\title{
Validation of Sustainability Criteria as a Tool for the Evaluation of Habitability of Prefabricated Concrete Homes for Andean Areas
}

\author{
Marcel Paredes ${ }^{1,2, *}$, Alexis Andrade ${ }^{1,3}$, Tito Castillo ${ }^{1}$, Valeria Arroba ${ }^{1}$, Emma Cevallos $^{1}$, Rafaela Viteri $^{2,4}$ \\ ${ }^{1}$ Faculty of Engineering, Universidad Nacional de Chimborazo, Riobamba, 060150, Chimborazo, Ecuador \\ ${ }^{2}$ Higher Technical School of Industrial Engineers, Polytechnic University of Madrid, 28006, Madrid, Spain \\ ${ }^{3}$ Higher Technical School of Engineering of Roads, Canals and Ports, Polytechnic University of Valencia, 46022, Valencia, Spain \\ ${ }^{4}$ Higher Polytechnic School of Chimborazo, Science Faculty, Riobamba, 060150, Chimborazo, Ecuador
}

Received September 24, 2021; Revised November 23, 2021; Accepted December 13, 2021

\begin{abstract}
Cite This Paper in the following Citation Styles
(a): [1] Marcel Paredes, Alexis Andrade, Tito Castillo, Valeria Arroba, Emma Cevallos, Rafaela Viteri , "Validation of Sustainability Criteria as a Tool for the Evaluation of Habitability of Prefabricated Concrete Homes for Andean Areas," Civil Engineering and Architecture, Vol. 10, No. 1, pp. 152 - 162, 2022. DOI: 10.13189/cea.2022.100114.
\end{abstract}

(b): Marcel Paredes, Alexis Andrade, Tito Castillo, Valeria Arroba, Emma Cevallos, Rafaela Viteri (2022). Validation of Sustainability Criteria as a Tool for the Evaluation of Habitability of Prefabricated Concrete Homes for Andean Areas. Civil Engineering and Architecture, 10(1), 152 - 162. DOI: 10.13189/cea.2022.100114.

Copyright $\odot 2022$ by authors, all rights reserved. Authors agree that this article remains permanently open access under the terms of the Creative Commons Attribution License 4.0 International License

\begin{abstract}
The incorporation of sustainable projects in the construction field and the use of prefabricated products allow to provide solutions for the real estate industry due to low cost. The goal of this research is to confirm the sustainability criteria of three different certifications such as: BREEAM, LEED and VERDE. Making paired comparisons based on the AHP method relating according to priorities of importance, preference or probability of pairs of the elements, based each time on a represented criteria in the decision hierarchy. According to the calculated values and the results, the AHP process was used to evaluate certification methods. The results of weighting between the three certification methods, determined that LEED adjusts to the reality of the Andean zone of Ecuador. As this area is a fragile place and susceptible to the contamination of water, soil and ecosystems, the innovation presented in this method, when ranking and comparing the weightings, makes it have greater weight compared to the other two analyzed. In this way, it was possible to interpret and develop a table of importance of suitable criteria to be implemented in the Andean zone, having as a result that the most important criterion is water with a percentage of $40.43 \%$.
\end{abstract}

Keywords Sustainable Construction, Environmental Impact, Prefabricated, BREEAM, LEED, VERDE

\section{Introduction}

Construction is an activity that accelerates climate change and the depletion of natural resources, causing a significant environmental burden throughout its useful life, from its construction, occupation and demolition, through the use phase and that varies considerably depending on the type of building and its location [1]. In this way, the growing construction in urban areas causes a significant impact on the environment, the economy, and public health [2]; this economic sector is responsible for $40-50 \%$ of all energy use, doubling its consumption between 1973-2012 [3], and has also increased anthropogenic greenhouse gas emissions [4]. The incentive of a sustainable construction is the step of traditional construction, looking for alternatives in the construction and managing to reduce the resources, which will help with the protection and care of the environment creating a healthy environment [5].

The construction system is going through an important change, one of them is the construction of prefabricated houses [6], which has a significant growth in recent years, since it generates advantages such as its cost, elaboration times, custom adaptability, energy efficiency, location and respect to the environment [7]. In this context, it is necessary to know the systems of environmental energy certifications, to assess their structure and obtain the criteria 
that fit the Ecuadorian reality. In addition, it is important to mention that this research is part of a research project that looks to supply a tool for environmental certifications to prefabricated homes in Ecuador.

Currently are several certifications whose objective, apart from qualifying all types of constructions, is to make people aware of the importance of building in a sustainable and energy efficient way, since this generates benefits both for the environment and for the tenants themselves, lower consumption of water, electricity, gas consumption. Among the most applied in Latin America are the BREEAM and LEED certificates, while green, being a Spanish certification for the language, is important for Latin America.

\section{Materials and Methods}

Currently there are several international methodologies that allow to evaluate, classify and certify the degree of sustainability of the construction. Bibliographic research will allow to figure out diverse ways of classifying with different criteria to be able to be adapted in a better way to the Andean areas of Ecuador.

This section sets up the differences between the three international certification methodologies and how the sustainability evaluation criteria under the AHP method can be included in the case of the Andean areas of Ecuador. The Hierarchical Analysis (AHP) process allowed to obtain the best categories that were considered for prefabricated concrete housing projects in Andean areas. With the Evaluation of the categories, the application of the AHP method, a final category was obtained result by multiplying categories with alternatives by their own categories with their criteria [8].

The process requires the decision maker to provide subjective assessments about the relative importance of each criterion and then specify each of the decision alternatives and for each criterion [9]. This criterion included qualitative aspects, deciding in an efficient and graphic way an organization of the information in relation to the problem, decomposing it and analyzing it in parts [10]. Figure 1 shows the analysis of the methods for qualifying sustainable criteria.

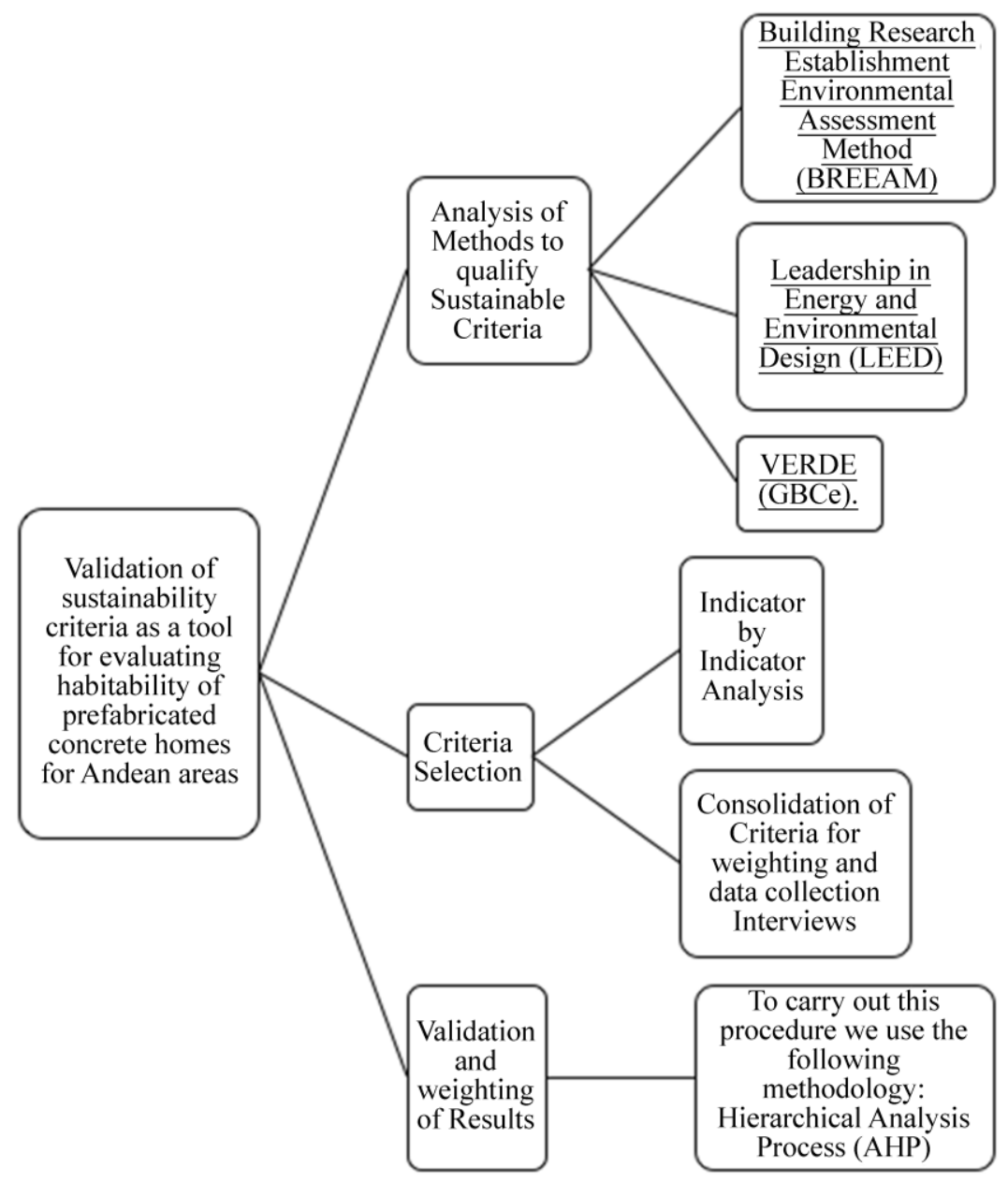

Figure 1. Analysis of methods to qualify sustainable criteria 


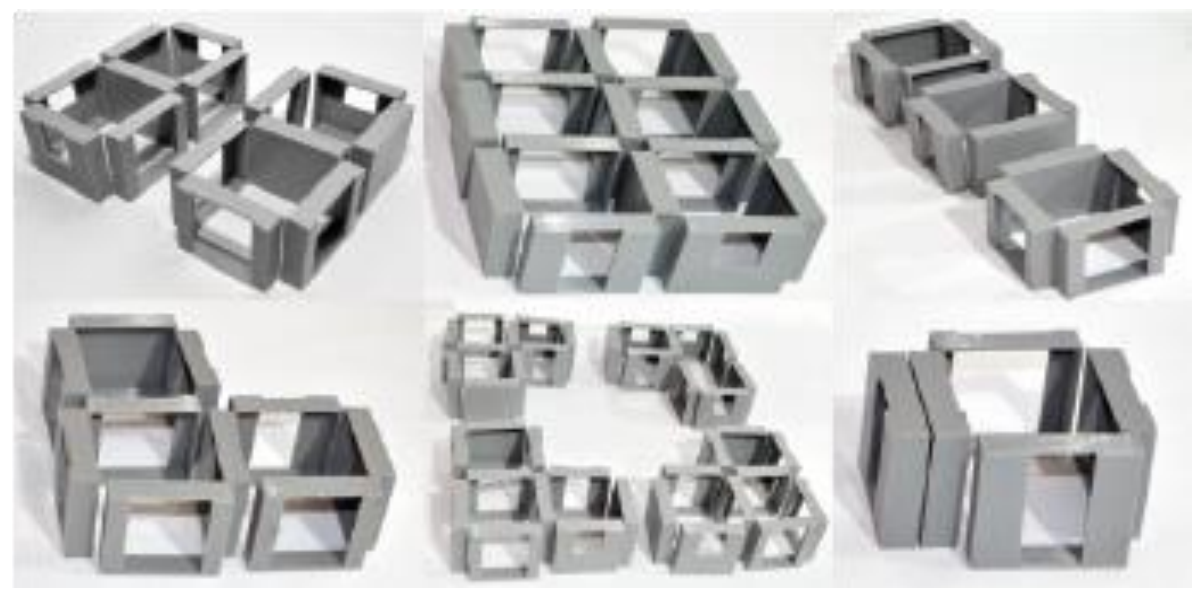

Figure 2. Type of manufactured home

Table 1. Saaty scale for AHP method

\begin{tabular}{|c|c|c|}
\hline Value & Definition & Comments \\
\hline 1 & Equal Importance & Criterion A is just as important as Criterion B \\
\hline 3 & Moderated Importance & Experience and judgment slightly favor criterion A over criterion B \\
\hline 5 & Great Importance & Experience and judgment strongly favor criterion A over B \\
\hline 7 & Greater Importance & Criterion A is much more important than Criterion B \\
\hline 9 & Extremely Important & The greater importance of criterion A over criterion B is beyond doubt \\
\hline $2,4,6$ y 8 & \multicolumn{2}{|c|}{ Intermediate values between the above when it is necessary to qualify. } \\
\hline
\end{tabular}

The main strategy of the AHP is paired comparisons, it uses an underlying scale with values from 1 to 9 to qualify the relative preferences of the elements, that is, a comparison is made between elements to find the order of the alternatives [11] as represented in table 1 .

For the case study we used the prototype of a house built with the prefabricated module of drawer Type Concrete of the Unión Cementera Nacional that contributed with the information in this investigation, The Figure 2 indicates the type of home.

The prioritization of the importance, preferences or probabilities of pairs of elements; depending on a common attribute or criterion represented in the decision hierarchy is the mathematical basis of the AHP [12].

In terms of quality in making the final decision, the consistency of the judgments shown during the series of paired comparisons is important. A perfect consistency is difficult to achieve, since in almost any set of paired comparisons there will always be some inconsistency; for they are judgments made by human beings [13]. If the degree of consistency is acceptable, the decision process can be continued, if the degree of consistency is unacceptable, the decision maker should reconsider and possibly modify their judgments about paired comparisons before proceeding with the analysis [14]. To figure out if the criteria are evaluated well, the calculation of the CR consistency ratio is used, where the result of the criteria is compared with the table shown in Table 2.
Table 2. Maximum percentage of CR consistency

\begin{tabular}{|c|c|}
\hline Matrix Size & \% Max CR \\
\hline 3 & $5 \%$ \\
\hline 4 & $9 \%$ \\
\hline$>=5$ & $10 \%$ \\
\hline
\end{tabular}

Formulas used for calculating the CR consistency ratio

$$
\begin{gathered}
\mathrm{IC}=\left(\lambda \_\mathrm{p}-\mathrm{n}\right) /(\mathrm{n}-1) \\
\mathrm{IA}=\left(1,98^{*}(\mathrm{n}-2)\right) / \mathrm{n} \\
\mathrm{CR}=\mathrm{IC} / \mathrm{IA}
\end{gathered}
$$

IC: Consistency Index

IA: Random consistency

CR: Consistency ratio

n: matrix order

$\lambda \_$: Average Landa

\section{Results and Discussions}

\subsection{Comparison of Methodologies}

As described in the method, in the first place, three certification criteria were compared, to supply its origin, which international organization uses it, but mainly which analysis each one performs, in this sense it is seen that the 
method LEED considers more analysis parameters, making this method more sensitive than the other two. Table 3 finds which are the most important criteria and which are compared according to the current situation of Ecuador and its Andean areas, performing an analysis of the importance of criteria according to the certification methods, thus finding the following:

Table 3. Unified criteria for the 3 certification methods BREEAM, LEED y VERDE

\begin{tabular}{|c|c|l|}
\hline \multicolumn{2}{|c|}{ Criteria } & \multicolumn{1}{c|}{ Comment } \\
\hline 1 & Water & $\begin{array}{l}\text { Water consumption in } \\
\text { construction }\end{array}$ \\
\hline 2 & Management & $\begin{array}{l}\text { Housing construction } \\
\text { management }\end{array}$ \\
\hline 3 & Health \& Wellness & $\begin{array}{l}\text { Health and well-being of the } \\
\text { people who build the house }\end{array}$ \\
\hline 4 & Energy & Energy use for construction \\
\hline 5 & Transport & $\begin{array}{l}\text { Transport of construction } \\
\text { materials }\end{array}$ \\
\hline 6 & Innovation & $\begin{array}{l}\text { Innovation in housing } \\
\text { construction }\end{array}$ \\
\hline 8 & Materials & $\begin{array}{l}\text { Materials used in the } \\
\text { construction of housing }\end{array}$ \\
\hline 9 & Waste & Housing construction waste \\
\hline 10 & Land use ecology & Land use \\
\hline
\end{tabular}

Table 4 shows the assessment and hierarchy of the criteria for the 3 methods. As can be seen, there is an inclination over the water criterion, with respect to the rest of the criteria that has greater importance as well as the Green methodology for Ecuador [15], either by the effect of the use of the resource or by the waterproofing of the soil, since the construction of houses does not allow natural infiltration. On the other hand, it is important the materials that are used, since they are prefabricated, it reduces the conduction or extraction of materials in the construction area so it has a strong importance, another criterion to be considered is the use of the land and the ecology that is modified by the construction of this type of buildings as well as what is exposed in the research of sustainability of productive systems [16], since as described above they change the permeability of the soil and also modify the ecology around the building, since both soils and surrounding vegetation are removed. The rest of the criteria can be considered moderate or low since they do not drastically influence the areas where this type of construction is intended to be set up.

Below is the normalized matrix Table 5, in which the importance of the criteria and their weights was calculated. From this matrix the importance of the criteria in the weighting column is deduced as the most important criterion is water, then the materials are seen, and innovation is also important in the construction of this type of housing. The values calculated for $\mathrm{CR}$, with 10 criteria are: $\mathrm{CI}$ is equal to $0.36, \mathrm{RI}$ is equal to 1.58 and $\mathrm{CR}$ is equal to 0.22 , therefore, you can continue with the AHP process, and assess between certification methods, the results are shown in Table 6.

Table 4. Comparing Criteria

\begin{tabular}{|c|c|c|c|c|c|c|c|c|c|c|c|}
\hline & & & & & & RITER & MATR & & & & \\
\hline $\begin{array}{r}\text { COMPARISON } \\
\text { CRITERIA }\end{array}$ & & $\frac{\bar{\pi}}{i \pi}$ & 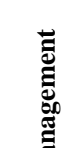 & 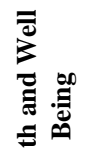 & 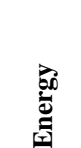 & $\begin{array}{l}\bar{\vdots} \\
\vdots \\
\bar{\vdots} \\
\bar{I}\end{array}$ & 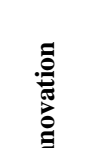 & 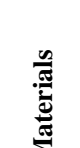 & 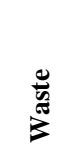 & 突 & 苞 \\
\hline & & 1 & 2 & 3 & 4 & 5 & 6 & 7 & 8 & 9 & 10 \\
\hline Water & 1 & 1 & 8 & 8 & 8 & 8 & 8 & 8 & 8 & 8 & 8 \\
\hline Management & 2 & $1 / 8$ & 1 & $1 / 5$ & $1 / 5$ & $1 / 3$ & $1 / 3$ & $1 / 5$ & $1 / 3$ & $1 / 3$ & 2 \\
\hline Health \& Wellness & 3 & $1 / 8$ & 5 & 1 & 2 & 2 & $1 / 3$ & $1 / 5$ & $1 / 3$ & $1 / 3$ & 2 \\
\hline Energy & 4 & $1 / 8$ & 5 & $1 / 2$ & 1 & $1 / 3$ & $1 / 3$ & $1 / 5$ & $1 / 3$ & $1 / 3$ & 2 \\
\hline Transport & 5 & $1 / 8$ & 3 & $1 / 2$ & 3 & 1 & 2 & $1 / 5$ & 2 & 2 & 2 \\
\hline Innovation & 6 & $1 / 8$ & 3 & 3 & 3 & $1 / 2$ & 1 & $1 / 5$ & 5 & 5 & 5 \\
\hline Materials & 7 & $1 / 8$ & 5 & 5 & 5 & 5 & 5 & 1 & 7 & 7 & 7 \\
\hline Waste & 8 & $1 / 8$ & 3 & 3 & 3 & $1 / 2$ & $1 / 5$ & $1 / 7$ & 1 & 5 & 5 \\
\hline Land use ecology & 9 & $1 / 8$ & 3 & 3 & 3 & $1 / 2$ & $1 / 5$ & $1 / 7$ & $1 / 5$ & 1 & 3 \\
\hline Contamination & 10 & $1 / 8$ & $1 / 2$ & $1 / 2$ & $1 / 2$ & $1 / 2$ & $1 / 5$ & $1 / 7$ & $1 / 5$ & $1 / 3$ & 1 \\
\hline Total & & 2.13 & 36.50 & 24.70 & 28.70 & 18.67 & 17.60 & 10.43 & 24.40 & 29.33 & 37.00 \\
\hline
\end{tabular}

Table 5. Standardized matrix of criteria 


\begin{tabular}{|c|c|c|c|c|c|c|c|c|c|c|c|c|}
\hline \multirow{3}{*}{$\begin{array}{l}\text { COMPARISON } \\
\text { OF CRITERIA }\end{array}$} & \multicolumn{10}{|c|}{ NORMALIZED MATRIX } & \multirow{3}{*}{ 䒘 } & \multirow{3}{*}{$\mathbf{A x P}$} \\
\hline & $\frac{\dot{\Xi}}{\tilde{J}}$ & 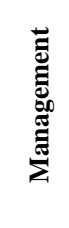 & 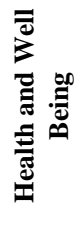 & 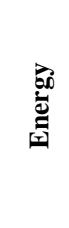 & 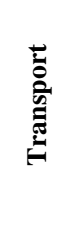 & 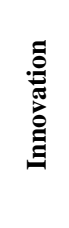 & 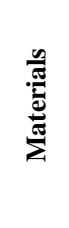 & $\begin{array}{l}\frac{\pi}{5} \\
\frac{5}{3}\end{array}$ & 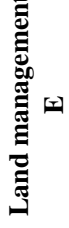 & $\stackrel{\Xi}{\stackrel{\Xi}{\Xi}}$ & & \\
\hline & 1 & 2 & 3 & 4 & 5 & 6 & 7 & 8 & 9 & 10 & & \\
\hline Water & 0.47 & 0.22 & 0.32 & 0.28 & 0.43 & 0.45 & 0.77 & 0.33 & 0.27 & 0.22 & 0.38 & 5.37 \\
\hline Management & 0.06 & 0.03 & 0.01 & 0.01 & 0.02 & 0.02 & 0.02 & 0.01 & 0.01 & 0.05 & 0.02 & 0.27 \\
\hline Health \& Wellness & 0.06 & 0.14 & 0.04 & 0.07 & 0.11 & 0.02 & 0.02 & 0.01 & 0.01 & 0.05 & 0.05 & 0.58 \\
\hline Energy & 0.06 & 0.14 & 0.02 & 0.03 & 0.02 & 0.02 & 0.02 & 0.01 & 0.01 & 0.05 & 0.04 & 0.41 \\
\hline Transport & 0.06 & 0.08 & 0.02 & 0.10 & 0.05 & 0.11 & 0.02 & 0.08 & 0.07 & 0.05 & 0.07 & 0.86 \\
\hline Innovation & 0.06 & 0.08 & 0.12 & 0.10 & 0.03 & 0.06 & 0.02 & 0.20 & 0.17 & 0.14 & 0.10 & 1.32 \\
\hline Materials & 0.06 & 0.14 & 0.20 & 0.17 & 0.27 & 0.28 & 0.10 & 0.29 & 0.24 & 0.19 & 0.19 & 2.70 \\
\hline Waste & 0.06 & 0.08 & 0.12 & 0.10 & 0.03 & 0.01 & 0.01 & 0.04 & 0.17 & 0.14 & 0.08 & 0.92 \\
\hline Land use ecology & 0.06 & 0.08 & 0.12 & 0.10 & 0.03 & 0.01 & 0.01 & 0.01 & 0.03 & 0.08 & 0.05 & 0.60 \\
\hline Contamination & 0.06 & 0.01 & 0.02 & 0.02 & 0.03 & 0.01 & 0.01 & 0.01 & 0.01 & 0.03 & 0.02 & 0.24 \\
\hline
\end{tabular}

Table 6. AHP between certification methods and by criteria

\begin{tabular}{|c|c|c|c|c|c|c|c|c|c|}
\hline \multicolumn{10}{|c|}{ WATER } \\
\hline \multirow{3}{*}{\multicolumn{2}{|c|}{$\begin{array}{l}\text { COMPARISON } \\
\text { OF CRITERIA }\end{array}$}} & \multicolumn{3}{|c|}{ CRITERIA MATRIX } & \multicolumn{3}{|c|}{ NORMALIZED MATRIX } & \multirow{3}{*}{ WEIGHTING } & \multirow{3}{*}{$\mathbf{A x P}$} \\
\hline & & $\begin{array}{c}\text { BREEAM } \\
\text { ES } \\
\end{array}$ & LEED & $\begin{array}{c}\text { VERDE } \\
\text { GBCe }\end{array}$ & $\begin{array}{c}\text { BREEAM } \\
\text { ES }\end{array}$ & LEED & $\begin{array}{c}\text { VERDE } \\
\text { GBCe }\end{array}$ & & \\
\hline & & 1 & 2 & 3 & 1 & 2 & 3 & & \\
\hline $\begin{array}{c}\text { BREEAM } \\
\text { ES }\end{array}$ & 1 & 1 & $1 / 5$ & $1 / 3$ & 0.11 & 0.13 & 0.08 & 0.11 & 0.32 \\
\hline LEED & 2 & 5 & 1 & 3 & 0.56 & 0.65 & 0.69 & 0.63 & 1.95 \\
\hline $\begin{array}{l}\text { VERDE } \\
\text { GBCe }\end{array}$ & 3 & 3 & $1 / 3$ & 1 & 0.33 & 0.22 & 0.23 & 0.26 & 0.79 \\
\hline \multicolumn{2}{|l|}{ TOTAL } & 9.00 & 1.53 & 4.33 & & & & No max & 3.06 \\
\hline \multicolumn{10}{|c|}{ MANAGEMENT } \\
\hline \multirow{3}{*}{\multicolumn{2}{|c|}{$\begin{array}{l}\text { COMPARISON } \\
\text { OF CRITERIA }\end{array}$}} & \multicolumn{3}{|c|}{ CRITERIA MATRIX } & \multicolumn{3}{|c|}{ NORMALIZED MATRIX } & \multirow{3}{*}{ WEIGHTING } & \multirow{3}{*}{$\mathbf{A x P}$} \\
\hline & & $\begin{array}{c}\text { BREEAM } \\
\text { ES } \\
\end{array}$ & LEED & $\begin{array}{c}\text { VERDE } \\
\text { GBCe }\end{array}$ & $\begin{array}{c}\text { BREEAM } \\
\text { ES } \\
\end{array}$ & LEED & $\begin{array}{c}\text { VERDE } \\
\text { GBCe }\end{array}$ & & \\
\hline & & 1 & 2 & 3 & 1 & 2 & 3 & & \\
\hline $\begin{array}{c}\text { BREEAM } \\
\text { ES }\end{array}$ & 1 & 1 & $1 / 8$ & 3 & 0.11 & 0.08 & 0.69 & 0.29 & 0.97 \\
\hline LEED & 2 & 8 & 1 & 2 & 0.89 & 0.65 & 0.46 & 0.67 & 3.42 \\
\hline $\begin{array}{l}\text { VERDE } \\
\text { GBCe }\end{array}$ & 3 & $1 / 3$ & $1 / 2$ & 1 & 0.04 & 0.33 & 0.23 & 0.20 & 0.63 \\
\hline \multicolumn{2}{|l|}{ TOTAL } & 9.33 & 1.63 & 6.00 & & & & No max & 5.03 \\
\hline \multicolumn{10}{|c|}{ HEALTH AND WELL BEING } \\
\hline \multirow{3}{*}{\multicolumn{2}{|c|}{$\begin{array}{l}\text { COMPARISON } \\
\text { OF CRITERIA }\end{array}$}} & \multicolumn{3}{|c|}{ CRITERIA MATRIX } & \multicolumn{3}{|c|}{ NORMALIZED MATRIX } & \multirow{3}{*}{ WEIGHTING } & \multirow{3}{*}{$\mathbf{A x P}$} \\
\hline & & $\begin{array}{c}\text { BREEAM } \\
\text { ES }\end{array}$ & LEED & $\begin{array}{c}\text { VERDE } \\
\text { GBCe } \\
\end{array}$ & $\begin{array}{c}\text { BREEAM } \\
\text { ES }\end{array}$ & LEED & $\begin{array}{l}\text { VERDE } \\
\text { GBCe }\end{array}$ & & \\
\hline & & 1 & 2 & 3 & 1 & 2 & 3 & & \\
\hline $\begin{array}{c}\text { BREEAM } \\
\text { ES }\end{array}$ & 1 & 1 & $1 / 4$ & $1 / 3$ & 0.11 & 0.16 & 0.08 & 0.12 & 0.35 \\
\hline LEED & 2 & 4 & 1 & 3 & 0.44 & 0.65 & 0.69 & 0.60 & 1.85 \\
\hline $\begin{array}{l}\text { VERDE } \\
\text { GBCe }\end{array}$ & 3 & 3 & $1 / 3$ & 1 & 0.33 & 0.22 & 0.23 & 0.26 & 0.81 \\
\hline TOTAL & & 8.00 & 1.58 & 4.33 & & & & No max & 3.01 \\
\hline
\end{tabular}


Table 6 Continued

\begin{tabular}{|c|c|c|c|c|c|c|c|c|c|}
\hline \multicolumn{10}{|c|}{ ENERGY } \\
\hline \multirow{3}{*}{\multicolumn{2}{|c|}{$\begin{array}{l}\text { COMPARISON } \\
\text { OF CRITERIA }\end{array}$}} & \multicolumn{3}{|c|}{ CRITERIA MATRIX } & \multicolumn{3}{|c|}{ NORMALIZED MATRIX } & \multirow{3}{*}{ WEIGHTING } & \multirow{3}{*}{$\mathbf{A x P}$} \\
\hline & & $\begin{array}{c}\text { BREEAM } \\
\text { ES }\end{array}$ & LEED & $\begin{array}{l}\text { VERDE } \\
\text { GBCe }\end{array}$ & $\begin{array}{l}\text { BREEAM } \\
\text { ES }\end{array}$ & LEED & $\begin{array}{l}\text { VERDE } \\
\text { GBCe }\end{array}$ & & \\
\hline & & 1 & 2 & 3 & 1 & 2 & 3 & & \\
\hline $\begin{array}{l}\text { BREEAM } \\
\text { ES }\end{array}$ & 1 & 1 & $1 / 3$ & $1 / 3$ & 0.11 & 0.22 & 0.08 & 0.14 & 0.37 \\
\hline LEED & 2 & 3 & 1 & 2 & 0.33 & 0.65 & 0.46 & 0.48 & 1.33 \\
\hline $\begin{array}{l}\text { VERDE } \\
\text { GBCe }\end{array}$ & 3 & 2 & $1 / 3$ & 1 & 0.22 & 0.22 & 0.23 & 0.22 & 0.65 \\
\hline \multicolumn{2}{|l|}{ TOTAL } & 6.00 & 1.67 & 3.33 & & & & No max & 2.36 \\
\hline \multicolumn{10}{|c|}{ TRANSPORT } \\
\hline \multirow{3}{*}{\multicolumn{2}{|c|}{$\begin{array}{l}\text { COMPARISON } \\
\text { OF CRITERIA }\end{array}$}} & CRI & ERIA M & RIX & NOI & LIZED & & \multirow{3}{*}{ WEIGHTING } & \multirow{3}{*}{$\mathbf{A x P}$} \\
\hline & & $\begin{array}{l}\text { BREEAM } \\
\text { ES }\end{array}$ & LEED & $\begin{array}{l}\text { VERDE } \\
\text { GBCe }\end{array}$ & $\begin{array}{c}\text { BREEAM } \\
\text { ES }\end{array}$ & LEED & $\begin{array}{l}\text { VERDE } \\
\text { GBCe }\end{array}$ & & \\
\hline & & 1 & 2 & 3 & 1 & 2 & 3 & & \\
\hline $\begin{array}{l}\text { BREEAM } \\
\text { ES }\end{array}$ & 1 & 1 & $1 / 4$ & $1 / 3$ & 0.11 & 0.16 & 0.08 & 0.12 & 0.35 \\
\hline LEED & 2 & 4 & 1 & 3 & 0.44 & 0.65 & 0.69 & 0.60 & 1.85 \\
\hline $\begin{array}{l}\text { VERDE } \\
\text { GBCe }\end{array}$ & 3 & 3 & $1 / 3$ & 1 & 0.33 & 0.22 & 0.23 & 0.26 & 0.81 \\
\hline \multicolumn{2}{|l|}{ TOTAL } & 8.00 & 1.58 & 4.33 & & & & No max & 3.01 \\
\hline \multicolumn{10}{|c|}{ INNOVATION } \\
\hline \multirow{3}{*}{\multicolumn{2}{|c|}{$\begin{array}{l}\text { COMPARISON } \\
\text { OF CRITERIA }\end{array}$}} & CRI & ERIA M & RIX & NOI & LIZED & & \multirow{3}{*}{ WEIGHTING } & \multirow{3}{*}{$\mathbf{A x P}$} \\
\hline & & $\begin{array}{c}\text { BREEAM } \\
\text { ES } \\
\end{array}$ & LEED & $\begin{array}{l}\text { VERDE } \\
\text { GBCe } \\
\end{array}$ & $\begin{array}{c}\text { BREEAM } \\
\text { ES } \\
\end{array}$ & LEED & $\begin{array}{c}\text { VERDE } \\
\text { GBCe } \\
\end{array}$ & & \\
\hline & & 1 & 2 & 3 & 1 & 2 & 3 & & \\
\hline $\begin{array}{l}\text { BREEAM } \\
\text { ES }\end{array}$ & 1 & 1 & $1 / 4$ & $1 / 3$ & 0.11 & 0.16 & 0.08 & 0.12 & 0.35 \\
\hline LEED & 2 & 4 & 1 & 3 & 0.44 & 0.65 & 0.69 & 0.60 & 1.85 \\
\hline $\begin{array}{l}\text { VERDE } \\
\text { GBCe }\end{array}$ & 3 & 3 & $1 / 3$ & 1 & 0.33 & 0.22 & 0.23 & 0.26 & 0.81 \\
\hline \multicolumn{2}{|l|}{ TOTAL } & 8.00 & 1.58 & 4.33 & & & & No max & 3.01 \\
\hline \multicolumn{10}{|c|}{ MATERIALS } \\
\hline \multirow{3}{*}{\multicolumn{2}{|c|}{$\begin{array}{l}\text { COMPARISON } \\
\text { OF CRITERIA }\end{array}$}} & CRI & ERIA M & RIX & NOI & LIZED & & \multirow{3}{*}{ WEIGHTING } & \multirow{3}{*}{$\mathbf{A x P}$} \\
\hline & & $\begin{array}{c}\text { BREEAM } \\
\text { ES }\end{array}$ & LEED & $\begin{array}{c}\text { VERDE } \\
\text { GBCe }\end{array}$ & $\begin{array}{c}\text { BREEAM } \\
\text { ES }\end{array}$ & LEED & $\begin{array}{l}\text { VERDE } \\
\text { GBCe }\end{array}$ & & \\
\hline & & 1 & 2 & 3 & 1 & 2 & 3 & & \\
\hline $\begin{array}{c}\text { BREEAM } \\
\text { ES }\end{array}$ & 1 & 1 & $1 / 5$ & $1 / 3$ & 0.11 & 0.13 & 0.08 & 0.11 & 0.32 \\
\hline LEED & 2 & 5 & 1 & 3 & 0.56 & 0.65 & 0.69 & 0.63 & 1.95 \\
\hline $\begin{array}{l}\text { VERDE } \\
\text { GBCe }\end{array}$ & 3 & 3 & $1 / 3$ & 1 & 0.33 & 0.22 & 0.23 & 0.26 & 0.79 \\
\hline \multicolumn{2}{|l|}{ TOTAL } & 9.00 & 1.53 & 4.33 & & & & No max & 3.06 \\
\hline \multicolumn{10}{|c|}{ WASTE } \\
\hline \multirow{3}{*}{\multicolumn{2}{|c|}{$\begin{array}{l}\text { COMPARISON } \\
\text { OF CRITERIA }\end{array}$}} & CRI & ERIA M & RIX & NOI & LIZED & & & \\
\hline & & $\begin{array}{c}\text { BREEAM } \\
\text { ES }\end{array}$ & LEED & $\begin{array}{l}\text { VERDE } \\
\text { GBCe }\end{array}$ & $\begin{array}{l}\text { BREEAM } \\
\text { ES }\end{array}$ & LEED & $\begin{array}{l}\text { VERDE } \\
\text { GBCe }\end{array}$ & WEIGHTING & $\mathbf{A x P}$ \\
\hline & & 1 & 2 & 3 & 1 & 2 & 3 & & \\
\hline $\begin{array}{l}\text { BREEAM } \\
\text { ES }\end{array}$ & 1 & 1 & $1 / 4$ & $1 / 3$ & 0.11 & 0.16 & 0.08 & 0.12 & 0.31 \\
\hline LEED & 2 & 3 & 1 & 2 & 0.33 & 0.65 & 0.46 & 0.48 & 1.28 \\
\hline $\begin{array}{l}\text { VERDE } \\
\text { GBCe }\end{array}$ & 3 & 2 & $1 / 3$ & 1 & 0.22 & 0.22 & 0.23 & 0.22 & 0.62 \\
\hline TOTAL & & 6.00 & 1.58 & 3.33 & & & & No max & 2.21 \\
\hline
\end{tabular}


Table 6 Continued

\begin{tabular}{|c|c|c|c|c|c|c|c|c|c|}
\hline \multicolumn{10}{|c|}{ USE OF LAND/ECOLOGY } \\
\hline \multirow{3}{*}{\multicolumn{2}{|c|}{$\begin{array}{l}\text { COMPARISON } \\
\text { OF CRITERIA }\end{array}$}} & \multicolumn{3}{|c|}{ CRITERIA MATRIX } & \multicolumn{3}{|c|}{ NORMALIZED MATRIX } & \multirow{3}{*}{ WEIGHTING } & \multirow{3}{*}{$\mathbf{A x P}$} \\
\hline & & $\begin{array}{c}\text { BREEAM } \\
\text { ES }\end{array}$ & LEED & $\begin{array}{l}\text { VERDE } \\
\text { GBCe }\end{array}$ & $\begin{array}{c}\text { BREEAM } \\
\text { ES }\end{array}$ & LEED & $\begin{array}{c}\text { VERDE } \\
\text { GBCe }\end{array}$ & & \\
\hline & & 1 & 2 & 3 & 1 & 2 & 3 & & \\
\hline $\begin{array}{c}\text { BREEAM } \\
\text { ES }\end{array}$ & 1 & 1 & $1 / 5$ & $1 / 3$ & 0.11 & 0.13 & 0.08 & 0.11 & 0.32 \\
\hline LEED & 2 & 5 & 1 & 3 & 0.56 & 0.65 & 0.69 & 0.63 & 1.95 \\
\hline $\begin{array}{l}\text { VERDE } \\
\text { GBCe }\end{array}$ & 3 & 3 & $1 / 3$ & 1 & 0.33 & 0.22 & 0.23 & 0.26 & 0.79 \\
\hline \multicolumn{2}{|l|}{ TOTAL } & 9.00 & 1.53 & 4.33 & & & & No max & 3.06 \\
\hline \multicolumn{10}{|c|}{ CONTAMINATION } \\
\hline \multirow{3}{*}{\multicolumn{2}{|c|}{$\begin{array}{l}\text { COMPARISON } \\
\text { OF CRITERIA }\end{array}$}} & \multicolumn{3}{|c|}{ CRITERIA MATRIX } & \multicolumn{3}{|c|}{ NORMALIZED MATRIX } & \multirow{3}{*}{ WEIGHTING } & \multirow{3}{*}{$\mathbf{A x P}$} \\
\hline & & $\begin{array}{c}\text { BREEAM } \\
\text { ES } \\
\end{array}$ & LEED & $\begin{array}{c}\text { VERDE } \\
\text { GBCe }\end{array}$ & $\begin{array}{c}\text { BREEAM } \\
\text { ES } \\
\end{array}$ & LEED & $\begin{array}{c}\text { VERDE } \\
\text { GBCe } \\
\end{array}$ & & \\
\hline & & 1 & 2 & 3 & 1 & 2 & 3 & & \\
\hline $\begin{array}{c}\text { BREEAM } \\
\text { ES }\end{array}$ & 1 & 1 & $1 / 5$ & $1 / 3$ & 0.11 & 0.13 & 0.08 & 0.11 & 0.32 \\
\hline LEED & 2 & 5 & 1 & 3 & 0.56 & 0.65 & 0.69 & 0.63 & 1.95 \\
\hline $\begin{array}{l}\text { VERDE } \\
\text { GBCe }\end{array}$ & 3 & 3 & $1 / 3$ & 1 & 0.33 & 0.22 & 0.23 & 0.26 & 0.79 \\
\hline \multicolumn{2}{|c|}{ TOTAL } & 9.00 & 1.53 & 4.33 & & & & No max & 3.06 \\
\hline
\end{tabular}

Table 7. Final decision of the appropriate certification method for the high Andean zone

\begin{tabular}{|c|c|c|c|c|c|c|c|c|c|c|c|c|}
\hline \multirow{3}{*}{\multicolumn{2}{|c|}{$\begin{array}{l} \\
\text { COMPARISON } \\
\text { OF CRITERIA }\end{array}$}} & \multicolumn{10}{|c|}{ CRITERIA MATRIX } & \multirow{3}{*}{ 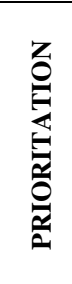 } \\
\hline & & \multirow{2}{*}{ 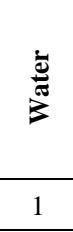 } & \multirow{2}{*}{ 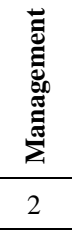 } & \multirow{2}{*}{ 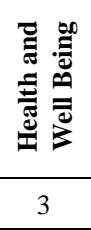 } & \multirow{2}{*}{ 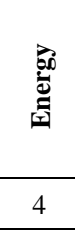 } & \multirow{2}{*}{ 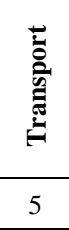 } & \multirow{2}{*}{ 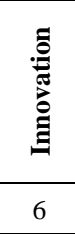 } & \multirow{2}{*}{ 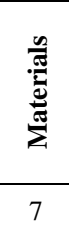 } & \multirow{2}{*}{ 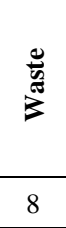 } & \multirow{2}{*}{ 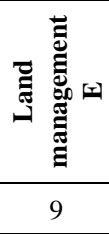 } & \multirow{2}{*}{ 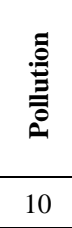 } & \\
\hline & & & & & & & & & & & & \\
\hline $\begin{array}{l}\text { BREEAM } \\
\text { ES }\end{array}$ & 1 & 0.32 & 0.97 & 0.35 & 0.37 & 0.35 & 0.35 & 0.32 & 0.31 & 0.32 & 0.32 & 4.52 \\
\hline LEED & 2 & 1.95 & 3.42 & 1.85 & 1.33 & 1.85 & 1.85 & 1.95 & 1.28 & 1.95 & 1.95 & 25.09 \\
\hline $\begin{array}{l}\text { VERDE } \\
\text { GBCe }\end{array}$ & 3 & 0.79 & 0.63 & 0.81 & 0.65 & 0.81 & 0.81 & 0.79 & 0.62 & 0.79 & 0.79 & 10.29 \\
\hline \multicolumn{2}{|c|}{ PONDERACIÓN } & 5.37 & 0.27 & 0.58 & 0.41 & 0.86 & 1.32 & 2.70 & 0.92 & 0.60 & 0.24 & \\
\hline
\end{tabular}

The analysis used in Table 6 is the process of weightings conducted through an average of the standardized criteria, what is analyzed later is a weighting by criterion according to the 3 methodologies of criteria obtaining a unique weighting which allows us to take into account the own weighting for each of the criteria such as water, contamination innovation, as analyzed in the research on sustainability criteria for the use of paints [17]. After subtracting these results, the final decision is made on the proper certification method that can be implemented in the upper Andean zone as shown in Table 7.

To make the final decision, it is important to use the weights obtained from Table 7, where also for the result of prioritization it is important to use the weighting obtained in Table 6 , in this way the respective calculation is made giving us as a result the prioritization of each of the criteria. In this sense, the final decision is presented, which is remade in the LEED certification method, since it has $25.09 \%$ of the final weighting with respect to the other methods the AHP model, determining that BREEAM shows that this criterion is the most adaptable for the reality and the current state of the high Andean zone in Ecuador in the same way as can be seen in the research carried out in Bogotá on the feasibility analysis of costs to implement LEED certification [18]. 
Table 8. Suitable criteria to be implemented in the Andean zone

\begin{tabular}{|c|c|c|c|}
\hline CRITERIA & CATEGORY & $\begin{array}{c}\text { SCORE BY } \\
\text { CATEGORY }\end{array}$ & TOTAL SCORE \\
\hline \multirow{4}{*}{ Water } & Reduction of water consumption & 8.55 & \multirow{4}{*}{40.43} \\
\hline & Water reuse and recycling & 18.56 & \\
\hline & Water meter at the level of the whole house & 8.44 & \\
\hline & Water meters during construction & 4.88 & \\
\hline \multirow{2}{*}{ Management } & Commissioning & 0.51 & \multirow{2}{*}{2.01} \\
\hline & Impact of the construction area & 1.5 & \\
\hline \multirow{4}{*}{ Health \& Wellness } & Natural lighting & 1.52 & \multirow{4}{*}{4.39} \\
\hline & Sound insulation & 0.9 & \\
\hline & Artificial lighting & 1.3 & \\
\hline & Private space of the house & 0.67 & \\
\hline \multirow{7}{*}{ Energy } & Minimum energy efficiency & 0.14 & \multirow{7}{*}{3.08} \\
\hline & Energy meter at the level of the whole house & 1.05 & \\
\hline & Improved reception & 0.12 & \\
\hline & Optimization of energy efficiency & 0.3 & \\
\hline & Renewable energy production & 0.68 & \\
\hline & Improved refrigerant management & 0.21 & \\
\hline & Green energy and carbon offsets & 0.58 & \\
\hline \multirow{7}{*}{ Transport } & Location of urban development & 1.7 & \multirow{7}{*}{6.51} \\
\hline & Sensitive soil protection & 0.95 & \\
\hline & Density of the environment and diverse uses & 0.57 & \\
\hline & Access to quality public transport & 1.23 & \\
\hline & Bicycle facilities & 0.88 & \\
\hline & Reduced parking footprint & 0.94 & \\
\hline & Sustainable vehicles & 0.24 & \\
\hline \multirow{2}{*}{ Innovation } & Innovation & 5.89 & \multirow{2}{*}{9.94} \\
\hline & Accredited professional & 4.05 & \\
\hline \multirow{8}{*}{ Materials and Resources } & Storage and collection of recyclables & 2.22 & \multirow{8}{*}{20.31} \\
\hline & Construction and demolition waste management planning & 3.79 & \\
\hline & Reducing the impact on the life cycle of housing & 2.31 & \\
\hline & Environmental product declarations & 1.28 & \\
\hline & Raw Material Sources & 2.8 & \\
\hline & Components of Materials & 2.31 & \\
\hline & Design for flexibility & 2.5 & \\
\hline & Construction and Demolition Waste Management & 3.1 & \\
\hline \multirow{4}{*}{ Waste } & Waste Management on site & 1.19 & \multirow{4}{*}{6.97} \\
\hline & Recycled aggregates & 1.37 & \\
\hline & Waste storage & 2.59 & \\
\hline & Waste composting & 1.82 & \\
\hline \multirow{4}{*}{ Land use Ecology } & Site selection & 0.69 & \multirow{4}{*}{4.56} \\
\hline & Protection of elements of ecological value & 1.6 & \\
\hline & Mitigation/improvement of ecological value & 1.49 & \\
\hline & Footprint of the house & 0.78 & \\
\hline \multirow{4}{*}{ Contamination } & Flood risk & 0.08 & \multirow{4}{*}{1.80} \\
\hline & Emissions of nitrogen oxides (Nox) & 0.05 & \\
\hline & Contamination of watercourses & 1.42 & \\
\hline & Reduction of external light pollution and noise pollution & 0.25 & \\
\hline
\end{tabular}




\subsection{Suitable Criteria to be implemented in the High Andean Zone}

Each Criterion was detailed with its categories and the most important ones are announced below:

\section{Water}

Reuse and recycling of water, this categorization is one of the most important and covers $18.56 \%$ of the total percentage that is $40.43 \%$, because through reuse allows us to innovate and preserve the care of water resources and their sustainability [19]. The proper use of water allows to reduce consumption and reuse in a responsible way each cubic meter of it allowing to reduce the cost of the work and its maintenance.

\section{Management}

Impact of the work area, this category obtains a score of $1.5 \%$, due to the importance of the place where this construction is going to be conducted and if it would affect or could cause any environmental impact to the area previously set up for the construction [20].

\section{Health and Well Being}

Natural Lighting, this category encompasses a reduction in electrical energy consumption, as it tries to use the most daily lighting. Thus, deciding its score of $1.52 \%$, being the most important of the criterion [21].

\section{Energy}

Energy Meter at the level of the entire house, this category covers $1.05 \%$, since its function is to distribute energy equitably in the different areas of a house, allowing to have an estimate of the energy consumption of each construction [22].

\section{Location and Transport}

Location of Urban Development, this category handles providing the necessary information to know the viability in terms of the construction of prefabricated homes, because with $1.7 \%$ it is possible to qualify if it is an urban or rural sector [23].

\section{Innovation}

Innovation, it is important to implement reduction systems that allow to control and reduce the number of resources that are generated during the useful life of the house. Implementing ecological strategies that allow a greater performance of this category that has a score of $5.89 \%$ [24].

\section{Materials and Resources}

Planning of Construction and Demolition Waste Management, it is important to have a clear idea of the use that will be given to the resources and waste generated in the planning and execution of the project. To give a correct rating to this category since its percentage is
$3.79 \%[25]$.

Waste

Waste storage, this type of storage allows us to conduct an adequate control, considering its importance of reuse and consumption for later projects inside or outside the house. Being important its rating of $2.59 \%$ [26].

\section{Land Use and Ecology}

Protection of elements of ecological value, it is important to consider the entire ecological and environmental environment within the construction and in this way give it the meaning and later care of it. The score of $1.60 \%$ will lie according to the care and maintenance of all the existing ecology inside and outside the house [27].

\section{Contamination}

Contamination of water courses, from the moment of the construction of the house it is important to have a flow of water that supplies us and provides the adequate amount of it for the projects in execution, but it is important to design possible treatments for reuse or release, without any type of pollution and in this way generate a sustainable construction taking into account the most important resource. Its score of $1.42 \%$ will be correct according to the maintenance given to it [28].

\section{Conclusions}

At present, Ecuadorian society shows the lack of potential of the models and tools that evaluate the sustainability of the construction of prefabricated housing, it is for this reason that this research project finds the BREEAM, LEED and VERDE GR models and certifications by comparing them and choosing the criteria that would adapt to the reality of the country, especially in the Ecuadorian Andean zone.

The comparison of certifications with each other was conducted, sharing most of the criteria that are related and allow a more thorough and practical analysis of the construction. The result of weighting between the three certification methods accredits the LEED method, in addition to adjusting to the reality of the Andean zone, because this area is a fragile place and susceptible to contamination of water, soil and ecosystems. The innovation presented by the LEED method, by hierarchizing and crossing the weights make it have greater weight with respect to the other two analyzed. The consideration of the AHP method allows us to deduce according to our criteria which would be the categories that could be adapted to our environment and social and economic reality in Ecuador.

For this reason, it was possible to interpret and develop a table of Importance of Criteria suitable to be implemented in the Andean zone in which through the 
values of Importance of Criteria between certification methods, it has been possible to interpret their criteria and give value to the categories, resulting in 10 criteria.

Criteria such as Water Efficiency has a percentage of 40.43\% among certification methods for homes in the Ecuadorian Andean zone, being the most important to be qualified within the criteria certification chart. Materials and resources occupy $20.31 \%$ of importance due to the difficulty of considering sources of environmentally friendly materials. Considering its categories such as, the reuse and recycling of water that will allow to use fewer resources and reduce water consumption, among others. According to research conducted, it was possible to decide that in Ecuador buildings are made with sustainable certifications, which allows considering several criteria such as Lighting, Energy, Water, among others.

\section{Acknowledgements}

We are especially grateful to the company Unión Cementera Nacional UCEM that supplied the information needed for the prefabricated elements.

\section{REFERENCES}

[1] Chakradeo U., Mohite D., "Lifecycle assessment methods and procedures and their role in measuring the sustainability component of a construction technology," Encycl. Renew. Sustain. Mater, vol. 1, pp. 158-166, 2020. DOI: 10.1016/b978-0-12-803581-8.11334-7

[2] Yang K, Sun W, Luo Y, Zhao L., "Impact of urban expansion on vegetation: The case of China (2000-2018)," J. Environ. Manage, vol. 291, p. 112598, 2021. DOI: 10.1016/j.jenvman.2021.112598

[3] Ahmed N, Abdel-Hamid M, Abd El-Razik M, El-Dash K, "Impact of sustainable design in the construction sector on climate change," Ain Shams Eng. J, vol. 12, no. 2, pp. 1375-1383, 2021. DOI: 10.1016/j.asej.2020.11.002

[4] Pereira L, Posen I, "Lifecycle greenhouse gas emissions from electricity in the province of Ontario at different temporal resolutions," J. Clean. Prod, vol. 270, p. 122514, 2020. DOI: $10.1016 /$ j.jclepro.2020.122514

[5] Ahmad W, Ahmad A, Ostrowski K, Aslam F, Joyklad P, “A scientometric review of waste material utilization in concrete for sustainable construction," Case Stud. Constr. Mater, vol. 15, p. e00683, 2021. DOI: 10.1016/j.cscm.202 1.e00683

[6] Lu W, Lee W, Xue F, Xu J, "Revisiting the effects of prefabrication on construction waste minimization: A quantitative study using bigger data," Resour. Conserv. Recycl, vol. 170, p. 105579, 2021. DOI: 10.1016/j.resconrec.2021.105579

[7] Tumminia G, Guarino F, Longo S, Ferraro M, Cellura M, Antonucci V, "Lifecycle energy performances and environmental impacts of a prefabricated building module," Renew. Sustain. Energy Rev, vol. 92, pp. 272-283, 2018. DOI: $10.1016 / J . R S E R .2018 .04 .059$

[8] Toskano H, Bruno G, "El proceso de análisis Jerárquico (AHP) como herramienta para la toma de decisiones en la construcción," Tesis digitales UNMSM, https://sisbib.unm sm.edu.pe (accessed Jul. 8, 2021.

[9] Hossen M, Kang S, Kim J, "Construction schedule delay risk assessment by using combined AHP-RII methodology for an international NPP project," Nucl. Eng. Technol, vol. 47, no. 3, pp. 362-379, 2015. DOI: 10.1016/J.NET.2014.1 2.019

[10] Taylan O, Bafail A, Abdulaal R, Kabli M, "Construction projects selection and risk assessment by fuzzy AHP and fuzzy TOPSIS methodologies," Appl. Soft Comput, vol. 17, pp. 105-116, 2014. DOI: 10.1016/J.ASOC.2014.01.003

[11] Coffey L, Claudio D, "In defense of group fuzzy AHP: A comparison of group fuzzy AHP and group AHP with confidence intervals," Expert Syst. Appl, vol. 178, p. 114970, 2021. DOI: 10.1016/J.ESWA.2021.114970

[12] Benítez J, Carpitella S, Certa A, Izquierdo J, "Management of uncertain pairwise comparisons in AHP through probabilistic concepts," Appl. Soft Comput, vol. 78, pp. 274-285, 2019. DOI: 10.1016/J.ASOC.2019.02.020

[13] Lin C, Kou G, "A heuristic method to rank the alternatives in the AHP synthesis," Appl. Soft Comput, vol. 100, p. 106916, 2021. DOI: 10.1016/J.ASOC.2020.106916

[14] Lima Junior F, Osiro L, Carpinetti L, "A comparison between Fuzzy AHP and Fuzzy TOPSIS methods to supplier selection," Appl. Soft Comput, vol. 21, pp. 194209, 2014. DOI: 10.1016/J.ASOC.2014.03.014

[15] Paredes M, Viteri R, "Certificación Energética «Verde» para Multiresidenciales en el Ecuador," Ciencias Medio Ambiente y Biodiversidad, 1 st ed, UNACH, pp. 15-25, 2018.

[16] Sanes Orrego A, "El análisis de ciclo de vida (ACV) en el desarrollo sostenible: propuesta metodológica para la evaluación de la sostenibilidad de sistemas productivos." Universidad Nacional de Colombia, https://repositorio.unal .edu.co (accessed Apr. 8, 2021).

[17] Rodriguez F, "Criterios de sostenibilidad en las certificaciones LEED, BREEAM y VERDE para el uso de pinturas, recubrimientos y TVOCs en edificación." Centro Universitario de la Defensa en la Escuela Naval Militar, http://calderon.cud.uvigo.es (accessed Jun. 21, 2021).

[18] Martínez M, Villalba D, Misle R, Rey E, Páez H, "Environmental and cost feasibility analysis when implementing LEED certification: A case study applied to an affordable housing project in Bogota D.C.," Rev. Ing. Constr, vol. 34, no. 1, pp. 99-110, 2019. DOI: 10.4067/s0718-50732019000100099

[19] Luo K, Scofield J, Qiu Y, "Water savings of LEED-certified buildings," Resour. Conserv. Recycl, vol. 175, p. 105856, 2021. DOI: 10.1016/J.RESCONREC.2021.105856

[20] Obata S, Agostinho F, Almeida C, Giannetti B, "LEED certification as booster for sustainable buildings: Insights for a Brazilian context,” Resour. Conserv. Recycl, vol. 145, 
pp. 170-178, 2019. DOI: 10.1016/J.RESCONREC. 2019.0 2.037

[21] Fakhari M, Fayaz R, Asadi S, "Lighting preferences in office spaces concerning the indoor thermal environment," Front. Archit. Res, vol. 10, no. 3, pp. 639-651, 2021. DOI: 10.1016/J.FOAR.2021.03.003

[22] Ferreira J, Pinheiro M, Brito J, "Portuguese sustainable construction assessment tools benchmarked with BREEAM and LEED: An energy analysis," Energy Build, vol. 69, pp. 451-463, 2014. DOI: 10.1016/J.ENBUILD.2013.11.039

[23] Cease B, Kim H, Kim D, Ko Y, Cappel C, "Barriers and incentives for sustainable urban development: An analysis of the adoption of LEED-ND projects," J. Environ. Manage, vol. 244, pp. 304-312, 2019. DOI: 10.1016/J.JENVMAN. 2019.04.020

[24] Pelli P, "Service innovation and sustainable construction: Analyses of wood vis-à-vis other construction projects," Clean. Eng. Technol, vol. 2, p. 100061, 2021. DOI: 10.1016/J.CLET.2021.100061
[25] Meneghelli A, "Whole-building embodied carbon of a North American LEED-certified library: Sensitivity analysis of the environmental impact of buildings materials," Build. Environ, vol. 134, pp. 230-241, 2018. DOI: 10.1016/J.BUILDENV.2018.02.044

[26] Chi B, Lu W, Ye M, Bao Z, Zhang X, "Construction waste minimization in green building: A comparative analysis of LEED-NC 2009 certified projects in the US and China," J. Clean. Prod, vol. 256, p. 120749, 2020. DOI: 10.1016/J.JCLEPRO.2020.120749

[27] Li Z, Luan W, Zhang Z, Su M, "Relationship between urban construction land expansion and population/economic growth in Liaoning Province, China," Land use policy, vol. 99, p. 105022, 2020. DOI: 10.1016/J.LANDUSEPOL.202 0.105022

[28] Gurgun A, Polat G, Damci A, Bayhan H, "Performance of LEED energy credit requirements in European countries," Procedia Eng, vol. 164, pp. 432-438, 2016. DOI: 10.1016/J.PROENG.2016.11.641 